\title{
Synthesis and Characterization of Polyacrylic Acid Based Nano-Hybrid Polymers for Malachite Green Uptake
}

\author{
Ashish Kumar1,2, G.S. Chauhan ${ }^{2, *}$ \\ ${ }^{1}$ Department of Chemistry, Government PG College, Chamba - 176 314, Himachal Pradesh, India. \\ ${ }^{2}$ Department of Chemistry, Himachal Pradesh University, Summer Hill Shimla - 171 005, Himachal Pradesh, India.
}

\section{A R T I C L E D E T A I L S}

Article history:

Received 26 July 2020

Accepted 18 August 2020

Available online 25 August 2020

\section{Keywords:}

Nano-hybrid

Malachite Green

MRC

\begin{abstract}
A B S T RA C T
Removal of malachite green from water bodies is an environmental concern of utmost priority. It requires adsorbents that can efficiently operate under real conditions. In view of this, a new polyacrylic acid based nano-hybrid polymer was synthesized by using sol-gel method in which acrylic acid acts as an organic part and tetraethoxysilane (TEOS) as an inorganic component. The new series of two other nano-hybrid polymers were also synthesized by using nanoparticles of iron oxide and titanium dioxide. The synthesized nano-hybrid polymers were extensively characterized using FTIR and XRD spectroscopic techniques. Further the synthesized nano-hybrid polymers were subjected to swelling studies with respect to different parameter such as time, temperature and pH. Malachite green was used as a cationic dye for studying the uptake behavior of synthesized polymers. Maximum retention capacity (MRC) and reusability of hybrid polymers were also evaluated up to ten cycles. The hybrid polymer, poly(AAc)/TEOS was more effective with high dye retention capacity.
\end{abstract}

\section{Introduction}

Dyes in waste water forms a huge challenge as their discharge contributes to around $\sim 20 \%$ of water pollution [1]. These are used in many industries and a huge load of $\sim 7 \times 10^{4}$ tonnes/year is released in environment [2]. Malachite green is an organic compound which is used as a dye stuff and has emerged as a contentious agent in aqua culture used for farming of aquatic organism, such as fish, crustaceans, molluscs and aquatic plants and used as a dye for materials such as silk, leather and paper [3]. In 1992, Canadian authorities declared that eating fish contaminated with malachite green pose a major health risk [4]. Malachite green was classified a Class II health hazard. In June 2007, the FDA blocked the importation of several varieties of seafood due to continued malachite green contamination [4]. Discharge of large amount of MG in water not only deteriorates the quality of freshwater, but seriously affects biodiversity and health of ecosystems by inhibiting sunlight reaching the aquatic flora and fauna thus reducing photosynthesis and increases biological oxygen demand [5]. Diverse chemical and physical methods have been published for dye removal from wastewaters [6], but most of the reported methods have major drawbacks including low efficiency, high cost and produce extra wastes. Hence, researchers focused on to develop more efficient, eco-friendly and economic nano-hybrid polymer. Nanohybrid polymers are a new class of organic materials. The word hybrid means "a mongrel" a compound formed by mixing of two different components [7]. Recently, considerable reports have been published on the synthesis of organic/inorganic nano-hybrid polymers. The aim of hybrid synthesis is to incorporate the properties of both materials/components, such as flexibility, toughness and easy processing of the organic component and hardness, strength and thermal stability of the inorganic part into a single entity. Under suitable conditions, the hybrid could exhibit better optical, electronic, optoelectronic, magnetic, mechanical and thermal properties than any of its precursor materials/components [8-12]. Hybrids have become important materials in many fields such as drug delivery, biomaterials or electronics. Assembly of molecular or polymeric species of organic and inorganic nature through interactions on the nano-scale constitutes the basis for the research of monohybrids materials. The development of these materials represents an emerging and inter-disciplinary issue at the border of sciences, material sciences and nanotechnology. They are of great interest due to their versatile applications in important areas as diverse as regenerative medicine and new materials with improved functional and structural properties [13-15]. Due to the flexible mode of synthesis and modification, various hybrid materials having wide applications were synthesized using sol-gel method in the past few years [16]. This present work describes the synthesis of a new hybrid polymer [poly(AAc) /TEOS] and its precursors $\left[\mathrm{Poly}(\mathrm{AAc}) / \mathrm{TEOS} / \mathrm{NaOH}, \quad\right.$ poly(AAc) $/ \mathrm{TEOS} / \mathrm{TiO}_{2}$ and poly(AAc) /TEOS/ $\mathrm{Fe}_{2} \mathrm{O}_{3}$ ] using sol-gel method [16]. Swelling behavior of the hybrid polymers were also accomplished. The hybrid polymer and its precursors were further subjected for its end-use for the uptake of an organic dye, malachite green. Maximum retention capacity and reusability of the nanohybrid polymer and its precursors were also evaluated.

\section{Experimental Methods}

\subsection{Chemicals}

Acrylic acid (AAc, Aldrich), tetraethoxysilane (TEOS, J\&K Chemika, 95\%) sodium dodecylsulphate (SDS, Beijing Chemical Plant), $\mathrm{HCl}$ (36 wt. $\%$ ), $n$-hexane (Beijing Chemical Plant), pH tablet (Himedia. Mumbai) of analytical grade were used. The double distilled water was used throughout this study.

\subsection{Procedure for Nano-Hybrid Synthesis}

Synthesis of poly(AAc)-based nano-hybrid polymer was accomplished in two stages. In the first stage, $10.0 \mathrm{~g}$ of TEOS was dissolved in $20 \mathrm{~mL} n$ hexane and $0.1 \mathrm{~mL}$ of $\mathrm{HCl}$ ( $5 \%$ by weight) was added as a catalyst. The contents were kept for vigorous stirring about $10 \mathrm{~min}$ at room temperature. In the second stage, $70 \mathrm{~mL}$ of $n$-hexane, $25 \mathrm{~mL}$ of deionized water and $16 \mathrm{~mL}$ of $\mathrm{HCl}$ was used to form the oil/water emulsion using sodium dodecyl sulphate (SDS, $75 \mathrm{mg}$ ) as a surfactant. To this reaction mixture $10 \mathrm{wt} \%$ of AAc ( $2.5 \mathrm{~g}$ ) was added. The contents were vigorously stirred for 30 min. Finally, the emulsion formed content of stage two was transferred to the mixture of the first stage. The mixture was aged at $37^{\circ} \mathrm{C}$ for $120 \mathrm{hr}$; the solid formed was separated from the supernatant. After continuous washing with acetone, methanol and water, the separated solid was kept in vacuum at $100^{\circ} \mathrm{C}$ for $50 \mathrm{hr}$. 


\subsection{Procedure for Functionalization of Nano-Hybrid}

The synthesized poly(AAc)-based nano-hybrid polymer has the composition of poly(AAc)/TEOS which was further functionalized by reaction with dilute $\mathrm{NaOH}(0.1 \%)$. The resultant product was designated as poly(AAc) $/ \mathrm{TEOS} / \mathrm{Na}^{+}$. Two other nano-hybrid polymers were also synthesized by in-situ loading of $2.5 \mathrm{~g}$ of $\mathrm{Fe}_{2} \mathrm{O}_{3}$ nanoparticles and $\mathrm{TiO}_{2}$ using the above discussed method. The resultant hybrid materials were designated as: poly(AAc)/TEOS/ $/ \mathrm{Fe}_{2} \mathrm{O}_{3}$ and poly(AAc)/TEOS/TiO 2 .

\subsection{Characterization of Nano-Hybrid Polymers}

The synthesized nano-hybrid polymers were extensively characterized using FTIR spectroscopy and XRD to revealed the evidence of hybrid formation. FTIR spectra of synthesized hybrid polymers were recorded on Nicolette 5700 in transmittance mode KBr. X-ray diffraction patterns of hybrid polymers were recorded on PANAnalytic XRD with XPERT-PRO diffractometer system using a typical wavelength of $1-54060 \AA(\mathrm{Cu}-\mathrm{K} \alpha$ radiation). The diffraction angle $2 \theta$ was varied from $10-70^{\circ}$.

\subsection{Swelling Behavior of Nano-Hybrid Polymers}

Swelling behavior of the hybrid polymers were evaluated as a function of time $(0-45 \mathrm{~min})$, temperature and $\mathrm{pH}$. A known weight $(0.1 \mathrm{~g})$ of the respective hybrid polymer was used for swelling studies. The temperature sensitivity of the hybrid polymers was determined by swelling these at various temperatures $\left(27,32,37\right.$ or $\left.42^{\circ} \mathrm{C}\right)$ for $8 \mathrm{hr}$. The $\mathrm{pH}$ study at the values $2.0,4.0,7.0,9.2$ and 12.0 were done at $37^{\circ} \mathrm{C}$. The swollen hybrid polymer was taken out from the solution, wiped-off softly with tissue paper and weighed immediately to calculate \% swelling $\left(\mathrm{P}_{\mathrm{s}}\right)$. $\mathrm{P}_{\mathrm{s}}$ was calculated as follows:

$$
\mathrm{P}_{\mathrm{s}}=\frac{\text { Weight of swollen hybrid-weight of dry hybrid }}{\mathrm{Weight} \text { of dry hybrid }} \times 100
$$

\subsection{Evaluation of Malachite Green Dye Uptake by Hybrid Polymers}

The standard curve for malachite green dye was prepared by preparing solution in the concentration range of $0-20 \mathrm{ppm}$. The optical density of the dye solution was measured on 6600 UV/VIS spectrophotometer. For the dye uptake $20 \mathrm{mg}$ of the respective hybrid polymer was immersed in a 50 $\mathrm{mL}$ of the prepared dye solution having concentration of $20 \mathrm{ppm}$. After the stipulated time, the polymer sample was removed from the solution and the optical density of the solution was measured on the UV/VIS spectrophotometer. The uptake studies were carried out as a function of time $(5-45 \mathrm{~min})$, temperature $\left(25-45{ }^{\circ} \mathrm{C}\right), \mathrm{pH}(2.0-12.0)$ and concentration (10-50 ppm). The \%uptake of the dye ( $\mathrm{Pu}_{\mathrm{u}}$ ) was calculated as follows:

$$
\mathrm{P}_{\mathrm{u}}=\mathrm{C}_{0}-\mathrm{C}_{t}
$$

Maximum retention capacity (MRC) was calculated by applying following equation:

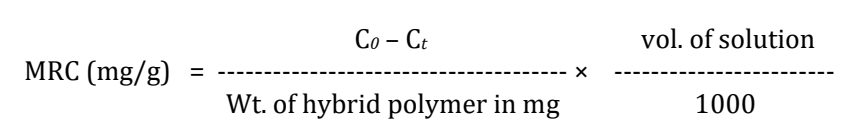

\section{Results and Discussion}

\subsection{Synthesis of Nano-Hybrid Polymers}

Sol-gel method was used for the synthesis of nano-hybrid polymers [16]. Tetraethylorthosilicate (TEOS) was taken as an inorganic component. The synthesized nano-hybrid polymers were listed as follows: a) Poly(AAc)/TEOS having Si-O-Si linkage (b) poly(AAc)/TEOS/TiO having $\mathrm{Si}-\mathrm{O}-\mathrm{Si}$ and having $\mathrm{Si}-\mathrm{O}-\mathrm{Ti}$ linkage (c) poly(AAc)/TEOS/ $/ \mathrm{Fe}_{2} \mathrm{O}_{3}$ having Si-O-Si linkage (d) poly(AAc)- $/$ TEOS/Na+.

\subsection{Characterization of Hybrid Polymers}

\subsubsection{Characterization using FTIR Spectroscopy}

Characterization of the synthesized hybrid polymers was carried out by FTIR spectroscopy. The characteristic absorption band of carboxylic group (C=0 stretching) was observed around $1742-1699 \mathrm{~cm}^{-1}$ for all hybrid polymers. From the FTIR spectrum of poly(AAc)/TEOS (Fig. 1) the hybrid formation was confirmed by Si-O-Si asymmetric stretching (1100-1052 $\left.\mathrm{cm}^{-1}\right)$ and symmetric stretching $\left(797 \mathrm{~cm}^{-1}\right)$. In the FTIR spectrum of poly(AAc) $/ \mathrm{TEOS} / \mathrm{TiO}_{2}$, a sharp peak was detected at $1721 \mathrm{~cm}^{-1}$ for https://doi.org/10.30799/jacs.225.20060202 carboxylic acid group ( $\mathrm{C}=0$ stretching). The hybrid polymer also showed a peak at 1500-1400 $\mathrm{cm}^{-1}$, indicating that some of the carboxylic acid group of poly(AAc) was chelated with $\mathrm{TiO}_{2}$. The strong absorption band in the range of $850-400 \mathrm{~cm}^{-1}$ in the FTIR spectrum corresponds to Ti-O-Ti network was also detected (Fig. 1). The band near $3400 \mathrm{~cm}^{-1}$ was characteristic band of residual $\mathrm{Ti}-\mathrm{OH}$ and $\mathrm{Si}-\mathrm{OH}$ groups in the hybrid polymers. From the FTIR spectrum of poly(AAc)/TEOS $/ \mathrm{Fe}_{2} \mathrm{O}_{3}$ hybrid formation was confirmed by Si-O-Si asymmetric and symmetric stretching peaks as discussed earlier and $\mathrm{Fe}-\mathrm{OH}$ peak in the FTIR spectrum at 3400 $\mathrm{cm}^{-1}$, while $\mathrm{C}=0$ stretching was reduces to $1637 \mathrm{~cm}^{-1}$.

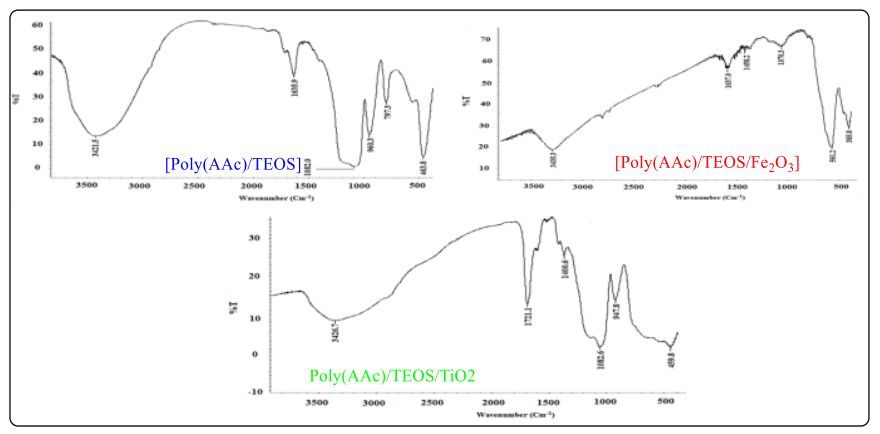

Fig. 1 FTIR spectrum of synthesized nano-hybrid polymer and their precursors

\subsubsection{XRD Characterization}

XRD pattern of the synthesized nano-hybrid polymers were presented in Fig. 2. The particle size of the synthesized hybrid polymers was found out to be in few $\mathrm{nm}$, revealed the strong evidence of the nano-hybrid formation. The particle size was calculated as $0.664,0.445$ and $0.466 \mathrm{~nm}$ for poly(AAc)/TEOS, poly(AAc)/TEOS/TiO 2 , poly(AAc)/TEOS $/ \mathrm{Fe}_{2} \mathrm{O}_{3}$, respectively. The peak position, full width at half maximum, (FWHM) $2 \theta, d$ spacing and \% relative intensity of XRD spectrum was $(10.5745,0.0984$, $8.3662,32.93$ ) observed for poly(AAc)/TEOS. The hybrid polymer, poly(AAc)/TEOS/TiO 2 , the peak position, (FWHM) $2 \theta, d$ spacing and $\%$ relative intensity of XRD spectrum was 24.9136, 0.3444, 3.57405 and 100 For the nano-hybrid polymer poly(AAc)/TEOS $/ \mathrm{Fe}_{2} \mathrm{O}_{3}$, the peak position, (FWHM) $2 \theta, d$ spacing and \% relative intensity of XRD spectrum was $10.1028,01476,8.7557$, and 100 . From the relative intensity peak, we observed that the nano-hybrid polymer poly(AAc)/TEOS, shows amorphous nature while other nano-hybrid polymers, poly(AAc)/TEOS $/ \mathrm{TiO}_{2}$ and poly(AAc)/TEOS $/ \mathrm{Fe}_{2} \mathrm{O}_{3}$ have displayed some crystalline nature (Fig. 2).

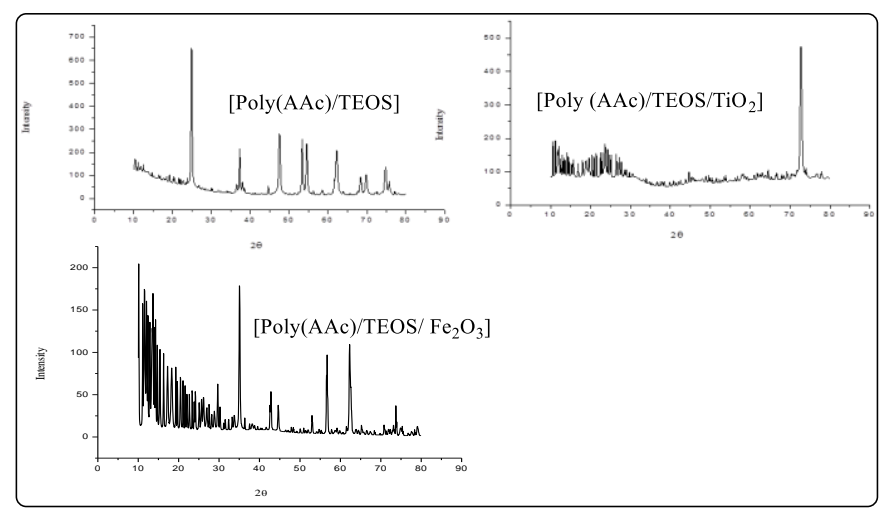

Fig. 2 XRD spectrum of synthesized nano-hybrid polymer and their precursors

\subsection{Swelling Behavior of Hybrid Polymers}

Swelling behavior studies of the synthesized nano-hybrid polymers is the most important aspect that defines their end-uses. The swelling behavior of nano-hybrid polymers is affected both by the structural and environmental factors. Taking cognizance of the same and the potential applications of the synthesized hybrid polymers, and their structure can be suitably tailored. The high and low swelling hybrid polymers have their respective merits in the fields where these find uses. The factors that are known to favor high swelling are osmotic potential, high free volume, high chain flexibility, low crosslinker density and strong interactions with water. The presence of hydrophilic or water solubilizing groups and ionic charges on the polymers are important structural factors, those affect maximization of water uptake. The swelling time, ionic strength of the aqueous solution are the environmental factors those affect the percent swelling $\left(\mathrm{P}_{\mathrm{s}}\right)$ of the hybrid polymers. Swelling studies of hybrid polymers help to establish structural and property relation and to know the effect of 
time, $\mathrm{pH}$, and temperature on hybrid polymers behavior. These studies also help to establish possible biomedical and pharmaceutical applications.

\subsubsection{Effect of Time and Temperature on Swelling Behavior}

An increase in the water uptake with an increase in time is the manifestation of the time dependent stretching of the crosslink chains of the hybrid polymers that allows more water in the interior of the hybrid. The swelling of synthesized hybrid polymers was studied at $20{ }^{\circ} \mathrm{C}$ with time variation from 5 to $45 \mathrm{~min}$ (Fig. 3). With an increase in swelling time the interaction of the functional sites with water molecules by weak forces (hydrogen bonding, Vander Waals, dipolar, hydrophilic and hydrophobic interactions) result in an increase in the size of pores, due to this flexibility of the chain increases and so does the amount of water retained by the hybrid polymers. It was observed that for the hybrid polymer, poly(AAc)/TEOS, maximum swelling took place among the four hybrid studied. The two other hybrid polymers which were synthesized by using iron oxide nanoparticles, i.e., poly(AAc)/TEOS $/ \mathrm{Fe}_{2} \mathrm{O}_{3}$ or poly(AAc)/TEOS $/ \mathrm{TiO}_{2}$ shows low $\mathrm{P}_{\mathrm{s}}$. It was observed that with increase in temperature, $\mathrm{P}_{\mathrm{s}}$ also increases for the hybrid polymers poly(AAc)/ TEOS and poly(AAc)/TEOS/NaOH , $\mathrm{P}_{\mathrm{s}}$ was maximum at $37{ }^{\circ} \mathrm{C}$ in the distilled water (Fig. 3). The two other hybrid polymers poly(AAc)/TEOS $/ \mathrm{Fe}_{2} \mathrm{O}_{3}$, poly(AAc)/ TEOS/ $\mathrm{TiO}_{2}$ showed maximum $\mathrm{P}_{\mathrm{s}}$ at temperature $42{ }^{\circ} \mathrm{C}$ in the distilled water. After $42{ }^{\circ} \mathrm{C}$ swelling decreases due to the elastic retractile nature of hybrid polymers and increase in the thermal mobility of water.

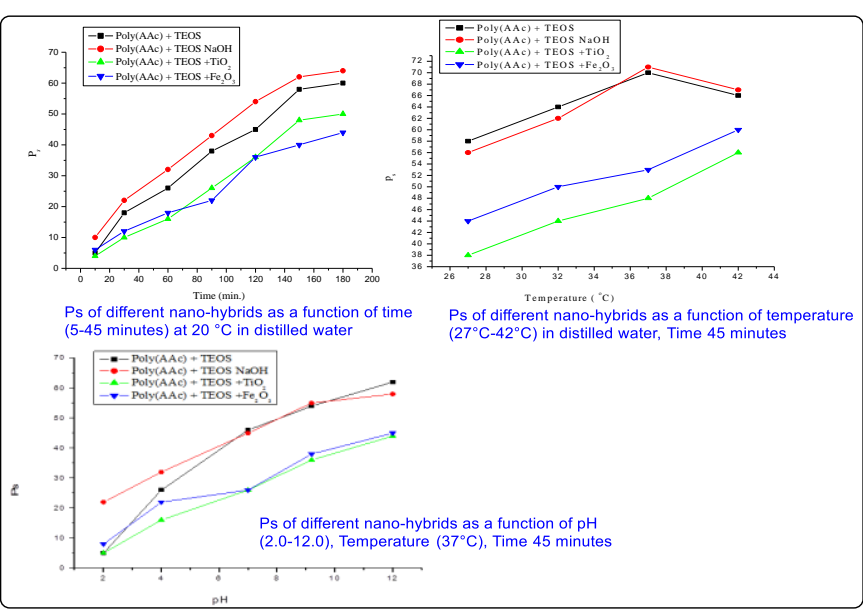

Fig. 3 Swelling behavior (Ps) of synthesized nano-hybrid polymer and their precursors

\subsubsection{Effect of pH on Swelling Behavior}

In this study, it has been observed that the hybrid polymer, poly(AAc)/TEOS show higher $\mathrm{P}_{\mathrm{s}}$ in alkaline $\mathrm{pH}$ than in acidic. At low $\mathrm{pH}$, a transient of hydrogen bonded complex in poly(AAc)/TEOS is formed due to the suppression of ionization of the carboxylic groups which makes water solubilizing groups less available for formation of hydrogen bonding with water. At the higher $\mathrm{pH}$ the carboxyl groups get ionized and results in high water uptake. Also, in the alkaline media the fully ionization of the carboxyl acid groups results in the repulsive forces within the matrix and leads to its expansion and higher swelling (Fig. 3).

\subsection{Uptake Study of Malachite Green Dye on Hybrid Polymers}

A standard curve of malachite green in the concentration range from 0 20 ppm was prepared by exploring graph between concentration of solution (ppm) and absorbance in UV spectrophotometer (Fig. 4). The absorbance wavelength used for malachite green in this study was $624 \mathrm{~nm}$. The following parameter i.e. time $(5-45 \mathrm{~min})$, temperature $\left(25-45^{\circ} \mathrm{C}\right), \mathrm{pH}$ (2.0-12.0) and concentration (10-50 ppm) were varied (Fig. 5). Maximum retention capacity (MRC) and reusability were studied up to10 cycles.

As comes out from Fig. 5, an increase in time increased the dye uptake. It reached the maximum after $45 \mathrm{~min}$. In case of temperature variation, uptake increases with increase in temperature and it was observed that at $37^{\circ} \mathrm{C}$, maximum $18.68 \%$ of malachite green uptake was observed (Fig. 5). Further increase in temperature decreases the uptake of dye. In the acidic $\mathrm{pH}$ solution, $\mathrm{P}_{\mathrm{u}}$ observed was low because at acidic $\mathrm{pH}$ the presence of unionized carboxyl group result in low swelling and low partitioning of dye to the hybrid particles. In the basic pH solutions at pH 9.2 and $12.0 \%$ uptake observed was $67 \%$ (Fig. 5). In the basic solution the occurrence of fully ionized acid groups increases repulsive forces within the matrix and leads to its expansion and higher swelling which leads to maximum $\mathrm{P}_{\mathrm{u}}$ of https://doi.org/10.30799/jacs.225.20060202 malachite green by nano-hybrid polymers. The uptake of malachite green has been increased with an increase in the dye concentration as shown in Fig. 5. A maximum $\mathrm{P}_{\mathrm{u}}(80 \%)$ was observed at $37^{\circ} \mathrm{C}$ when concentration of the dye solution was $50 \mathrm{ppm}$ and the $\mathrm{pH} 9.2$.

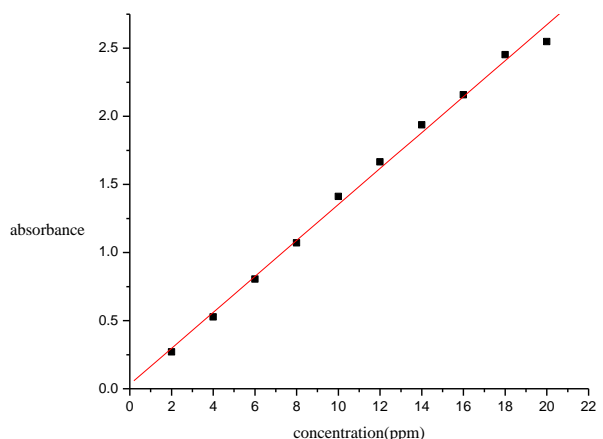

Fig. 4 Standard curve of malachite green

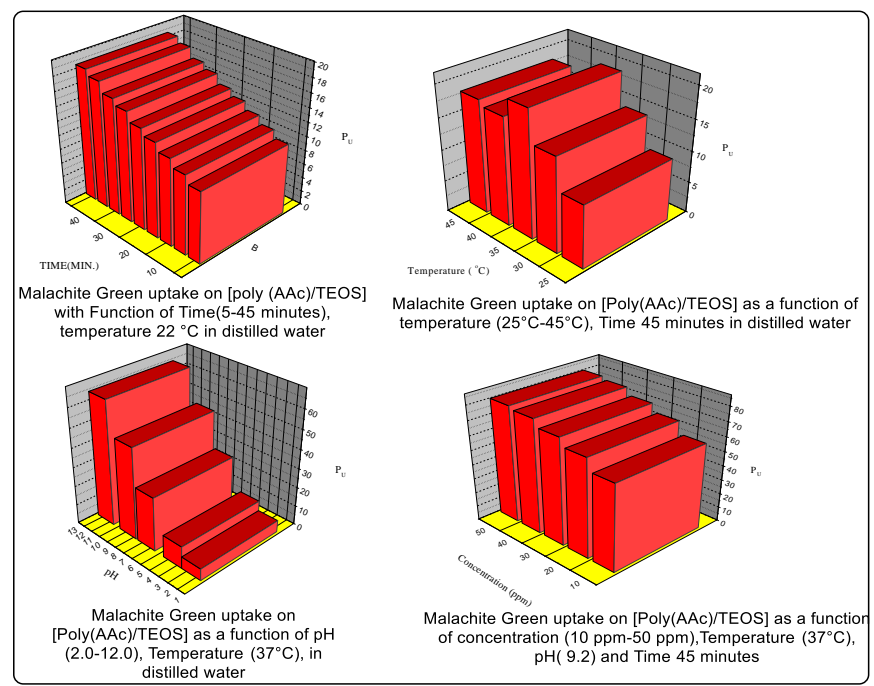

Fig. 5 Malachite green dye uptake on synthesized nano-hybrid polymer [poly(AAc)/TEOS]

\subsubsection{Maximum Retention Capacity and Reusability}

Maximum retention capacity (MRC) of the hybrid polymer is how much dye retained by it after successive adsorption or uptake cycles. MRC of the synthesized nano-hybrid polymer, poly(AAc)/TEOS was studied up to 10 cycles (Table 1). After this the polymer did not retain the dye and its dye retention capacity got saturated. The MRC of $9.598 \mathrm{mg} / \mathrm{g}$ was calculated for this nano-hybrid polymer. For the other hybrid polymers the MRC was found lower than poly(AAc)/TEOS (Table 1). Reusability for hybrid polymer, poly(AAc)/TEOS was studied up to10 cycles. Reusability is the reuse of the same sample for multiple times to absorb the dye from the solution. After first absorption the dye-loaded hybrid polymer was leached or washed with $0.5 \mathrm{~N} \mathrm{HCl}$ for reuse for number of times (up to 10 cycles) for dye uptake. The nano-hybrid polymer, poly(AAc)/TEOS (Fig. 6) showed maximum of $72 \%$ uptake up to $6^{\text {th }}$ cycles, after this the $\mathrm{P}_{\mathrm{u}}$ was reduced for next four cycles. The activity of hybrid polymer was reduced because of the consequent changes in the active site saturation; hence the substrate could not approach the active sites and result was a decrease in activity.

Table 1 Maximum Retention Capacity (MRC) of synthesized nano-hybrid polymer and their precursors

\begin{tabular}{llll}
\hline Cycle & Poly(AAc)/TEOS & Poly(AAc)/TEOS/TiO 2 & $\begin{array}{l}\text { Poly(AAc)/TEOS } \\
\text { treated with } \mathrm{NaOH}\end{array}$ \\
\hline 1 & 1.98 & 1.50 & 1.48 \\
2 & 1.72 & 1.31 & 1.29 \\
3 & 1.56 & 1.13 & 1.24 \\
4 & 1.42 & 0.98 & 0.97 \\
5 & 1.00 & 0.63 & 0.68 \\
6 & 0.92 & 0.47 & 0.41 \\
7 & 0.64 & 0.34 & 0.30 \\
8 & 0.23 & 0.22 & 0.25 \\
9 & 0.12 & 0.10 & 0.14 \\
10 & 0.00 & 0.00 & 0.00 \\
Total & 9.59 & 6.68 & 6.76 \\
\hline
\end{tabular}




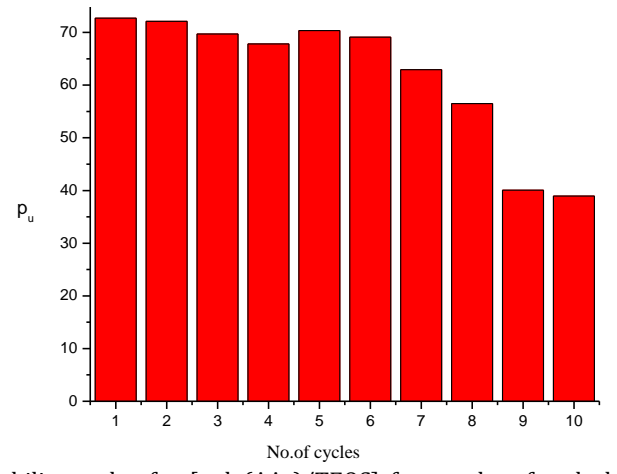

Fig. 6 Reusability cycles for [poly(AAc)/TEOS] for uptake of malachite green at temperature $\left(37^{\circ} \mathrm{C}\right)$, pH 9.2 and time 45 minutes

\section{Conclusion}

In this study a new nano-hybrid polymer and its precursor were synthesized as an active adsorbent for cationic dye, malachite green. The nano-hybrid polymers were synthesized by sol-gel method. These hybrid polymers were evaluated as adsorbents for malachite green. The hybrid polymer, poly(AAc)/TEOS was more effective with high dye retention capacity. Hence, it was further investigated at different adsorption conditions and the optimized conditions were found to be time $45 \mathrm{~min}$, temperature $37^{\circ} \mathrm{C}, \mathrm{pH} 9.2$ and concentration $10 \mathrm{ppm}$. The MRC of 9.598 $\mathrm{mg} / \mathrm{g}$ was calculated for this nano-hybrid polymer. The nano-hybrid polymer, poly(AAc)/TEOS showed $72 \%$ uptake up to $6^{\text {th }}$ cycles. Hence, it is appropriate alternative to the expensive adsorbents reported for the dye effluents of the wastewaters.

\section{Acknowledgements}

Authors acknowledge Dr. Rajeev Jindal, Associate Professor, NIT Jalandhar, for his support to carryout FTIR and XRD studies.

\section{References}

[1] R. Kant, Textile dyeing industry an environmental hazard, Nat. Sci. 4 (2012) 22-26.

[2] B. Noroozi, G.A. Sorial, H. Bahrami, M. Arami, Equilibrium and kinetic adsorption study of a cationic dye by a natural adsorbent-Silkworm pupa. J. Hazard. Mater. 139 (2006) 167-174.

[3] D.J. Alderman, Malachite green: a review. J. Fish Dis. 8 (1985) 289-298.

[4] S. Shivaji, R. Sinha, D. Roy, Toxicological effects of malachite green, Aquatic Toxicol. 66 (2004) 319-329.

[5] M.T. Yagub, T.K. Sen, S. Afroze, H.M. Ang, Dye and its removal from aqueous solution by adsorption: a review, Adv. Colloid Interf. Sci. 209 (2014) 172-184.

[6] S. Raghu, C.A. Basha, Chemical or electrochemical techniques, followed by ion exchange,for recycle or textile dye wastewater, J. Hazard. Mater. 149 (2007) 324-330.

[7] T. Ryo, Y. Chujo, Synthesis of IPN polymer hybrids of polystyrene gel and silica gel by an in-situ radical polymerization method, J. Mater. Chem. 8 (1998) 11131115 .

[8] W.J.E. Beek, R.A.J. Jansen, Photoinduced electron transfer in hetero supramolecular assemblies of $\mathrm{TiO}_{2}$ nanoparticles and terthiophene carboxylic acid in apolar solvents, Adv. Funct. Mater. 12 (2002) 519-525.

[9] E.J. Nassar, R.R. Gonvalves, M. Ferrai, Y. Messaddeq, J.L.R. Sidney, Titania-based organic-inorganic hybrid planar waveguides J. Alloys Compd. 344 (2002) 221225.

[10] S. Roux, G.J.A.A. SolerIllia, S. Demoustier-Champagne, P. Audebert, C. Sanchez, Titania/polypyrrole hybrid nanocomposites built from in-situ generated organically functionalized nanoanatase building blocks, Adv. Mater. 15 (2003) 217-221.

[11] P.C. Chiang, W.T. Whang, The synthesis and morphology characteristic study of BAO-ODPA polyimide/ $\mathrm{TiO}_{2}$ nano hybrid films, Polymer 44 (2003) 2249-2254

[12] R.L. Ballard, S.J. Tuman, D.J. Fouquette, W. Stegmiller, M.D. Soucek, Effects of an acid catalyst on the inorganic domain of inorganic- organic hybrid materials, Chem. Mater. 11 (1999) 726-735.

[13] E. Dujardin, S. Mann, Bio-inspired materials chemistry, Adv. Mater. 14 (2002) $775-788$.

[14] E. Ruiz-Hitzky, M. Darder, P. Aranda, Functional biopolymer nanocomposites based on layered solids, J. Mater. Chem.15 (2005) 3650-3662.

[15] M. Darder, P. Aranda, E. RuizHitzky, Bionanocomposites: a new concept of ecological, bioinspired, and functional hybrid materials, Adv. Mater. 19 (2007) 1309-1319.

[16] G. Philipp, H. Schmidt, New materials for contact lenses prepared from Si-and Ti-alkoxides by the sol-gel process, J. Non-Cryst. Solids 63 (1984) 283-292. 